\title{
Adaptive response of Pinus monticola driven by positive selection upon resistance gene analogs (RGAs) of the TIR-NBS-LRR subfamily
}

\author{
Antonios Zambounis ${ }^{(1)}$ \\ Evangelia Avramidou (2), \\ Aglaea Papadima ${ }^{(2)}$, \\ Athanasios Tsaftaris ${ }^{(1-3)}$, \\ lordanis Arzimanoglou ${ }^{(4)}$, \\ Evaggelos Barbas (2), \\ Panagiotis Madesis ${ }^{(3)}$ \\ Filippos A Aravanopoulos ${ }^{(2)}$
}

\begin{abstract}
Western white pine (Pinus monticola Dougl.) is an important forest tree species, which is intensively plagued by the fungus Cronartium ribicola. Resistance gene analogs (RGAs) are the most highly abundant class of potential resistance $(R)$ genes sharing greatly conserved domains and structures. Hence RGAs are crucial components for disease resistance breeding programs on $P$. monticola serving as useful functional markers. A total of $33 P$. monticola RGAs gene homologues were mined from GenBank, encoding for $R$ gene members of the TIR-NBS-LRR subfamily. The existence of positive selection acting upon RGAs was determined using a series of maximum likelihood analyses. Robust evidence of positive selection was showed to be acting widely in three clades across RGA gene phylogeny, both on terminal and ancestral lineages. Furthermore, our analysis revealed that the majority of positively selected residues sites are localized widely across these RGAs sequences, putatively affecting the structures of their ligand-binding domains and offering novel specificities. These results may find immediate application in ongoing disease resistance breeding programs.
\end{abstract}

Keywords: Fungal Diseases, Genomics-assisted Breeding, Non-synonymous Nucleotide Substitution, Positive Selection, Resistance Gene Analogs (RGAs), White Pines tium ribicola J.C. Fisch.). Trial results indicate large variation within provenance, growth comparable to that of local Abies borisii regis Mattf., but reduced survival (Papadima \& Sotikopoulos 1995, Panetsos et al. 1996).

Cronartium ribicola is among the most destructive white pine fungal diseases which has caused severe mortality in North American white pines and especially in $P$. monticola, which is currently considered a species under threat (Loehman et al. 2011). P. monticola species exhibits genetic variation in disease resistance to $C$. ribicola involving mainly a dominant disease resistance gene (Cr2 - Kinloch et al. 1999, Liu et al. 2006). Disease resistance and maintenance of ge-
(1) Laboratory of Genetics and Plant Breeding, Faculty of Agriculture, Forestry \& Natural Environment, Aristotle University of Thessaloniki, P.O. Box 261, Thessaloniki GR54124 (Greece); (2) Laboratory of Forest Genetics and Tree Breeding, Faculty of Agriculture, Forestry \& Natural Environment, Aristotle University of Thessaloniki, P.O. Box 238, Thessaloniki GR54006 (Greece); (3) Institute of Applied Biosciences, CERTH, Thermi, Thessaloniki, GR57001 (Greece); (4) Genomedica, Piraeus, GR18537 (Greece)

\section{@ Filippos A Aravanopoulos (aravanop@for.auth.gr)}

Received: Mar 09, 2016 - Accepted: Sep 27, 2016

Citation: Zambounis A, Avramidou E, Papadima A, Tsaftaris A, Arzimanoglou I, Barbas E, Madesis P, Aravanopoulos FA (2017). Adaptive response of Pinus monticola driven by positive selection upon resistance gene analogs (RGAs) of the TIR-NBS-LRR subfamily. iForest 10: 237 241. - doi: 10.3832/ifor2050-009 [online 2017-02-01]

Communicated by: Alberto Santini netic diversity in production populations are very important aspects for $P$. monticola breeding (Dangl et al. 2013). The development through breeding of trees resistant to fungi and the maintenance of a variety of genetic resources are of pivotal significance towards the substantial establishment of P. monticola reforestation and plantation forestry programs (Liu et al. 2004).

Classical breeding approaches, such as interspecific hybridization with the indigenous to Greece and resistant to C. ribicola Balkan white pine Pinus peuce Grisb., are time-consuming without warranties of specific results in a definite timeframe. Engagement of innovative molecular breeding approaches allows breeders to establish sustainable resistance more accurately and at a faster pace (Lalli et al. 2005). PCR approaches implementing mainly degenerate primers were extensively applied in order to isolate members of the NBS family (nucleotide-binding site) / LRRs (LeucineRich Repeats) - containing genes, the most highly expanded group of genes linked directly to resistance $(R)$ genes mediated functions in plants (Debener \& Byrne 2014). This protein family is further classified into two major subfamilies based on structural characteristics of their putative amino-terminal signaling domain (Kim et al. 2012). One subfamily, TIR-NBS-LRR, contains an amino-terminal signaling domain that depicts homology with Toll and interleukin- 1 receptor (TIR), whilst the other subfamily, CC-NBS-LRR, instead of the TIR domain, 
carries a putative coiled-coil structure (CC) one (Kim et al. 2012). Both these subfamilies have been reported to mediate resistance to a wide range of phytopathenic fungi (Wan et al. 2010).

Resistance gene analogs (RGAs) are a large class of potential R-genes, being an important resource for the development of molecular markers, hence enhancing numerous disease resistance breeding efforts (Ameline-Torregrosa et al. 2008, Perazzolli et al. 2014). Moreover, these RGAs commonly contain tandem repeats such as LRRs which give rise to novel ligand-binding specificities under positive selection (Khan et al. 2016) via a number of different modes, such as point mutations, variations in repeat numbers and gene duplications (Friedman \& Baker 2007, Meyers et al. 2003, Yang et al. 2008, Zambounis et al. 2012). Positive selection is defined as the rapid fixation of beneficial non-synonymous mutations and is an important evolutionary driving force for a number of paralogous genes towards their functional diversification, which might be selectively favored under biotic pressures in plants (Delph \& Kelly 2014). Furthermore, the location of positively selected amino acid residues is an important aspect for obtaining novel gene functions (MondragónPalomino et al. 2002). Previous studies have reported that solvent-exposed regions of the LRRs repeats are subjected to positive selection; such evidence has been interpreted as a sign of the involvement of these regions in pathogen recognition (Parniske et al. 1997, Zambounis et al. 2012, Khan et al. 2016). Overall, the evolution patterns of plant NBS-LRR $R$ genes and of their RGAs counterparts is likely a complex process (Zhou et al. 2004, Sekhwal et al. 2015).

The TIR-NBS-LRR RGAs from P. monticola were previously investigated for their diversity and expression, in order to gain insights regarding their potential usage in disease resistance breeding programs (Liu \& Ekramoddoullah 2003). In this study, we address the question whether signatures of positive selection are systematically employed as an evolutionary driven force acting on TIR-NBS-LRR-encoding RGAs genes at $P$. monticola. Thus, we gain insights into the evolutionary profiles of the $P$. monticola TIR-NBS-LRR-containing RGAs genes, hypothesizing that those successive episodes of positive selection might contribute in the acquisition of novel pathogens recognition repertoires. Overall, our findings enrich and support the hypothesis that these $P$. monticola RGAs could be employed as biomarkers for both, monitoring the ongoing and, more effectively applying future $P$. monticola disease resistance breeding efforts.

\section{Materials and methods}

P. monticola TIR-NBS-LRR RGAs mining and phylogenetic analysis

All 33 P. monticola RGAs partial proteins previously identified by Liu \& Ekramoddoullah (2003) were downloaded from the GenBank by keyword searches based on their NB-ARC domain profile (PFoo931.19/ CL0023). Using these amino acid sequence IDs, the respective ORF nucleotide sequences were also retrieved. All amino acid sequences had to fulfill the following criteria in older to be evaluated for evolutionary signatures acting upon them: (a) existence of valid structures by means of protein lengths up to 90 residues, positions of NBARC domain motifs, blast(p) hits against known plant resistance genes; and (b) verification of functional domains using the predictions against the Pfam database (http://pfam.sanger.ac.uk/search\#tabview= tab1) and the InterProScan 5 software (Jones et al. 2014).

The phylogenetic relationships among these P. monticola RGAs amino acid sequences were revealed by aligning them using the MUSCLE software (Edgar 2004) and performing a tree reconstruction using the RAxML software (Stamatakis 2014) inferring a gamma model of rate hetero-

Tab. 1 - Overview of CODEMLSITES method statistics and distribution of positively selected amino acid sites across TIR-NBS-LRR RGAs in P. monticola.

\begin{tabular}{|c|c|c|c|c|c|c|}
\hline Model & $\mathbf{P}$ & Parameters & $l$ & $\mathrm{dn} / \mathrm{ds}$ & $\begin{array}{l}\text { Positively } \\
\text { Selected } \\
\text { Sites }\end{array}$ & $\begin{array}{l}\text { Statistical } \\
\text { significance }\end{array}$ \\
\hline $\begin{array}{l}\text { MO: one } \\
\text { ratio }\end{array}$ & 2 & $\omega=0.395$ & -2855.76 & $=\omega$ & - & - \\
\hline M1: neutral & 3 & $\begin{array}{l}\mathrm{p}_{0}=0.72, \omega_{0}=0.22 \\
\mathrm{p}_{1}=0.27, \omega_{1}=1\end{array}$ & -2821.47 & 0.44 & - & - \\
\hline $\begin{array}{l}\text { M2: } \\
\text { selection }\end{array}$ & 5 & $\begin{array}{l}\mathrm{p}_{0}=0.72, \omega_{0}=0.22 \\
\mathrm{p}_{1}=0.177, \omega_{1}=1 \\
\mathrm{p}_{2}=0.102, \omega_{2}=1\end{array}$ & -2821.47 & 0.44 & - & $P<0.001$ \\
\hline $\begin{array}{l}\text { M3: } \\
\text { discrete }\end{array}$ & 6 & $\begin{array}{l}\mathrm{p}_{0}=0.201 \omega_{0}=0.048 \\
\mathrm{p}_{1}=0.594, \omega_{1}=0.335 \\
\mathrm{p}_{2}=0.197, \omega_{2}=1.019\end{array}$ & -2811.41 & 0.41 & $\begin{array}{c}19,50,56 \\
69,74,79 \\
83\end{array}$ & $P<0.001$ \\
\hline M7: beta & 3 & $p=0.825, q=1.26$ & -2812.75 & 0.39 & - & - \\
\hline $\begin{array}{l}\text { M8: beta } \\
\text { and } \omega\end{array}$ & 5 & $\begin{array}{l}p_{0}=0.923, p=1.164 \\
q=2.28 \\
p_{1}=0.076, \omega=1.322\end{array}$ & -2817.16 & 0.41 & $\begin{array}{l}19,56,69 \\
74,79,83\end{array}$ & $P<0.05$ \\
\hline
\end{tabular}

geneity. All these analyses were performed on the Geneious R7 platform (Kearse et al. 2012).

\section{P. monticola TIR-NBS-LRR RGAs \\ evolutionary analysis}

The YNOo, CODEML and CODEMLSITES programs were employed from the PAML version 4.8 software package (Yang 2007) in order to assess evidence of positive selection signatures acting in these $P$. monticola TIR-NBS-LRR RGAs. These tests compare the substitution rates of non-synonymous $(\mathrm{dN})$ to synonymous $(\mathrm{dS})$ mutations, and estimate the derived omega $(\omega)$ values, both among the nucleotide codon sequences and along the branch lineages. The rates of $d N / d S$ nucleotide substitutions per site across all possible pairwise sequence comparisons were estimated using the counting approach (Yang \& Nielsen 2000) implemented in the YNoo program. The total $d N, d S$, and $\omega$ values for all pairwise sequence comparisons among these RGAs are shown in Tab. S1 (Supplementary material).

An NJ tree at amino acid level and the respective nucleotide alignment were both used in the CODEML and CODEMLSITES analyses according to Lynn et al. (2004a, 2004b); details of the exact methods for these analyses are found in Zambounis et al. (2012). Briefly, the amino acid sequence alignment was conducted by employing the MUSCLE software (Edgar 2004), whilst the construction of the respective phylogenetic tree was performed using the MEGA 5 software (Tamura et al. 2011). A Poisson correction was employed with a gamma distribution modeling among sites and rates variations (Zambounis et al. 2012). Finally, the Baseml software (PAML version 4.8 - Yang 2007) was implemented for estimating the alpha shape parameter using the REV model.

CODEML was tested in P. monticola TIRNBS-LRR RGAs dataset for estimating the variable selective pressures acting among lineages in the phylogeny, whilst CODEMLSITES was employed to test for site-specific codon substitution models allowing the identification of selective signatures across amino acid residues (Tab. 1). In both programs log-likelihood calculations were performed for each model and comparisons between each other were implemented by likelihood ratio tests (LRTs). The empirical Bayesian method further allowed the detection of codon residues subjected to positive selection by higher posterior probabilities. All data sets as well as the outputs of CODEML and CODEMLSITES programs are available upon request.

\section{Results}

\section{P. monticola TIR-NBS-LRR RGAs} structure and phylogenetic analysis

The 33 P. monticola TIR-NBS-LRR RGAs studied were found to share Pfam hits with the NB-ARC domain (PFoo931.19) and the 


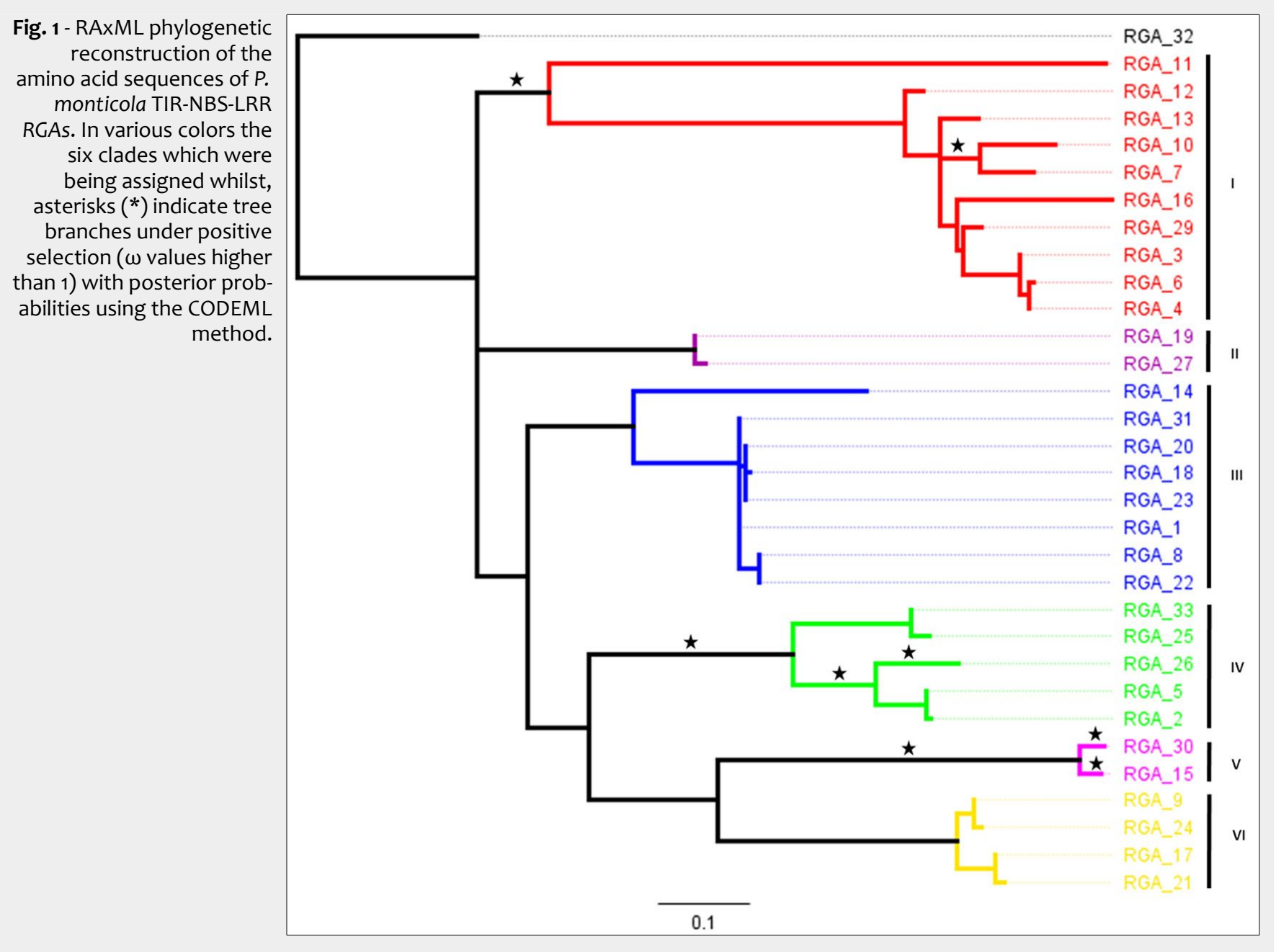

InterProScan ones with LRR-containing proteins (PTHR23155). The RGAs were corresponding to regions between conserved kinase-2 and P-loop domains. A RAxML phylogenetical analysis at the amino acid level was conducted in order to explore their expansion (Fig. 1). The backbone topology of this tree was properly resolved with 63 branches (Fig. 1), whilst the overall pairwise identity among the amino acid sequences was $59.6 \%$, indicative of a high degree of divergence.

\section{Positive selection acting across $P$.} monticola TIR-NBS-LRR RGAs lineages

In order to verify whether accelerated evolution and signs of positive selection might have also contributed to the divergence of these TIR-NBS-LRR RGAs genes, our RGAs dataset was subjected to several successive tests of positive selection using the counting (YNoo) and the ML (CODEML) CODEMLSITES) methods. A robust and reliable nucleotide alignment resulted, based on the presence of zero or few gaps across the aligned sequences. The $\mathrm{dN}$ and $\mathrm{dS} \mathrm{mu}$ tation values across the entire RGAs sequences were calculated using the counting YNoo method (see Tab. S1 in Supplementary material). Any substitution saturation effects were checked by calculating $\mathrm{dS}$ rates between the aligned nucleotide se- quences. The average numbers of synonymous (dS) mutations per synonymous sites over all sequence pairs were below the value of 2 , the crucial threshold value above which the corresponding sequences would have to be excluded from further evolutionary analyses in order to bypass any saturation effects based on nucleotide substitutions (Yang \& Nielsen 2000).

Finally, the CODEML approach was employed in order to identify branches subjected to positive selection. Statistically significant evidence of positive selection ( $\omega>$ 1.00) was detected in numerous branches (Fig. 1) with posterior probabilities $P<$ 0.0001 . These signs were found across clades I, IV and V acting with a non-general rule, as positive episodes were evidenced both in the ancient and terminal branches (reflecting probably a series of recently successive bursts of gene duplications).

\section{Positive selective pressures acting}

among amino acid residues across the P. monticola TIR-NBS-LRR RGAs

Possible positive selection at the individual amino acid residues level was investigated by the CODEMLSITES method (Yang 2007) which compared various models (Mo against $M_{3}, M_{1}$ against $M_{2}$, and finally $M_{7}$ against the stringent $M 8$ ) in our RGAs dataset. Two models ( $\mathrm{M}_{3}$ and $\mathrm{M} 8$ ) revealed positive selective pressures acting among amino acid residues under significant posterior probabilities $(P<0.0001$ and $P<0.05$, respectively - Tab. 1$)$. These extensive signs of positive selection were found acting rather widely upon amino acid residues and across the respective tree branches. The accurate locations of these positively selected sites (with $\omega$ values up to 1.48) are depicted in Tab. 1.

\section{Discussion}

Conifer genomes are in general large with DNA content reaching more than 26,500 Mbp per haploid genome (Aravanopoulos 2014 and references therein). Taking also into consideration their long generation times, classical breeding, especially for disease resistance is a very slow process. On the contrary, molecular breeding approaches may provide promising and effective strategies for both $R$ genes and RGAs counterpart identification based on their highly conserved motifs (Liu \& Ekramoddoullah 2007). Previously, it was postulated that there is a highly diverse group of TIRNBS-LRR genes in P. monticola (Liu \& Ekramoddoullah 2003) along with the existence of numerous CC-NBS-LRR-containing proteins (Liu \& Ekramoddoullah 2007). Similar abundance and diversity of these genes was also observed in other plant genomes 
(Zhou et al. 2004, Chen et al. 2010, Sekhwal et al. 2015). Hitherto, TIR-NBS-LRR RGAs in $P$. monticola exhibited high variation at amino acid levels, whilst almost half of them were found to be pseudogenes rep resenting a rather complex TIR-NBS-LRR subfamily (Liu \& Ekramoddoullah 2003).

In our study, the analysis of $33 \mathrm{P}$. monticola TIR-NBS-LRR RGAs showed a high level of diversity that may contribute to their evolutionary fitness, and the putatively functional character of their transcription activity (Liu \& Ekramoddoullah 2003). These RGAs are a potential source for the development of molecular markers, especially regarding selection of resistance to white pine blister rust. Furthermore, RGAs were found to share conserved structures rendering them ideal candidates for applying evolutionary bioinformatics approaches.

The CODEML analysis, showed recent episodes of positive selection acting extensively in clades I, IV and V, which in turn were overlapping similar more ancient events. Similar positive selective pressure acting on plant RGAs has also been reported in other surveys ( $\mathrm{Li}$ et al. 2006, Wan et al. 2010, Perazzolli et al. 2014, Khan et al. 2016). In principle, this reservoir of genetic variation could drive the evolution of new R-gene specificities (Perazzolli et al. 2014, Sekhwal et al. 2015). To our knowledge this is the first systematically report of evolutionary pressures acting in $P$. monticola RGAs. Positively selected RGAs branches were not only at terminal positions, implying rather recent positive selective signatures, but also at ancestral positions on the phylogeny. This finding is in accordance with previous reports which showed that numerous selective episodes occur even from the birth of a paralogous locus (Yang et al. 2008, Zambounis et al. 2012).

Signs of positively selected residues were found to be distributed rather evenly across the RGAs amino acid sequences. Positive selection on NBS-containing gene families in plants and especially among their LRR domains, were also reported in various plant hosts (Chen et al. 2010, Li et al. 2010, Zambounis et al. 2012, Yang et al. 2013, Perazzolli et al. 2014, Khan et al. 2016). These findings support the view that selection for durable disease resistance may be a crucial component of plant breeding programs. Additionally, comparative analyses of Rosaceae trees regarding their $R$ genes revealed that solvent-exposed residues of the LRRs domains are hyper-variable with intensive positive selective pressures acting on them (Perazzolli et al. 2014). Moreover, such evidence is consistent with host-pathogen co-evolution processes leading to the acquisition of novel resistance functional specificities (Zambounis et al. 2012, Perazzolli et al. 2014). These findings would facilitate the development of informative RGA-derived molecular markers for identifying fungal resistant $P$. monticola and $P$. monticola $\times P$. peuce genotypes in breeding programs using segregating progenies. They may also prove to be of importance for $P$. peuce itself, as despite the fact that Balkan white pine is a resistant species, future selection pressures on C. ribicola in light of climatic change may alter the virulence of the fungus towards its host.

\section{Conclusions}

The $P$. monticola RGAs gene homologues encoding for $R$ gene members of the TIRNBS-LRR subfamily were found to be subjected to intensive positive selection, acting widely in three clades across their phylogeny, both on terminal and ancestral lineages. The majority of positively selected residues sites were found to be localized widely across these RGAs sequences, putatively affecting the structures of their ligand-binding domains. Practical applications in the development of disease resistant plants through breeding applications may also be considered, facilitating for instance, the development of informative RGAsderived sequence characterized amplified region (SCAR) markers, based on RGAs sequences with extensive positive selection acting upon them. Such markers might be particularly useful for identifying fungal resistant genotypes in routine marker-assisted selection (MAS) breeding programs.

\section{List of Abbreviations}

The following abbreviations have been used throughout the paper:

CC: N-terminal coiled-coil domain;

- LRR: Leucine Rich Repeat;

- NBS: nucleotide-binding site domain;

- PAML: Phylogenetic Analysis by Maximum Likelihood;

- RaxML: Randomized Axelerated Maximum Likelihood;

- RGA: Resistance Gene Analog;

- TIR: Toll/interleukin-1 receptor domain;

-WWP: Western white pine.

\section{Acknowledgments}

The co-authors have not a conflict of interest to declare.

\section{References}

Ameline-Torregrosa C, Wang B-B, O'Bleness MS, Deshpande S, Zhu H, Roe B, Young ND, Cannon SB (2008). Identification and characterization of nucleotide-binding site-leucine-rich repeat genes in the model plant Medicago truncatula. Plant Physiology 146 (1): 5-21. - doi: 10.1104/pp. 107.104588

Aravanopoulos FA (2014). Genomics of trees. In: "Tree Biotechnology" (Ramawat KG, Merillon JM, Ahuja MR eds). CRC Press, Boca Raton, USA, pp. 514-557. - doi: 10.1201/b16714-23

Chen Q, Han Z, Jiang H, Tian D, Yang S (2010). Strong positive selection drives rapid diversification of R-genes in Arabidopsis relatives. Journal of Molecular Evolution 70 (2): 137-148. - doi: 10.1007/s00239-009-9316-4

Dangl JL, Horvath DM, Staskawicz BJ (2013). Pivoting the plant immune system from dissection to deployment. Science 341 (6147): 746-751. doi: $10.1126 /$ science.1236011

Debener T, Byrne DH (2014). Disease resistance breeding in rose: current status and potential of biotechnological tools. Plant Science 228: 107-117. - doi: 10.1016/j.plantsci.2014.04.005

Delph LF, Kelly JK (2014). On the importance of balancing selection in plants. New Phytologist 201 (1): 45-56. - doi: 10.1111/nph.12441

Edgar RC (2004). MUSCLE: a multiple sequence alignment method with reduced time and space complexity. BMC Bioinformatics 5: 1-19. doi: 10.1186/1471-2105-5-113

Friedman AR, Baker BJ (2007). The evolution of resistance genes in multi-protein plant resistance systems. Current Opinion in Genetics and Development 17 (6): 493-499. - doi: 10.1016/ j.gde.2007.08.014

Jones $\mathrm{P}$, Binns $\mathrm{D}$, Chang HY, Fraser $\mathrm{M}$, Li $\mathrm{W}$, McAnulla C, McWilliam H, Maslen J, Mitchell A, Nuka G, Pesseat S, Quinn AF, Sangrador-Vegas A, Scheremetjew M, Yong SY, Lopez R, Hunter $S$ (2014). InterProScan 5: genome-scale protein function classification. Bioinformatics 30 (9): 1236-1240. - doi: 10.1093/bioinformatics/btu031 Kearse M, Moir R, Wilson A, Stones-Havas S, Cheung $M$, Sturrock $S$, Buxton $S$, Cooper $A$, Markowitz S, Duran C, Thierer T, Ashton B, Mentjies P, Drummond A (2012). Geneious Basic: an integrated and extendable desktop software platform for the organization and analysis of sequence data. Bioinformatics 28 (12): 1647-1649. - doi: 10.1093/bioinformatics/bt S199

Khan AM, Khan AA, Azhar MT, Amrao L, Cheema HM (2016). Comparative analysis of resistance gene analogues encoding NBS-LRR domains in cotton. Journal of the Science of Food and Agriculture 96 (2): 530-538. - doi: 10.1002/jsfa. 7120

Kinloch BB, Sniezko RA, Barnes GD, Greathouse TE (1999). A major gene for resistance to white pine blister rust in western white pine from the western Cascade Range. Phytopathology 89: 861-867. - doi: 10.1094/PHYTO.1999.89.10.861 Kim J, Lim CJ, Lee BW, Choi JP, Oh SK, Ahmad R, Kwon SY, Ahn J, Hur CG (2012). A genome-wide comparison of NB-LRR type of resistance gene analogs (RGA) in the plant kingdom. Molecules and Cells 33 (4): 385-392. - doi: 10.1007/s10059012-0003-8

Lalli DA, Decroocq V, Blenda AV, Schurdi-Levraud V, Garay L, Le Gall O, Damsteegt V, Reighard GL, Abbott AG (2005). Identification and mapping of resistance gene analogs (RGAs) in Prunus: a resistance map for Prunus. Theoretical and Applied Genetics 111 (8): 1504-1513. - doi: 10.1007/s00122-005-0079-z

Li J, Xu Y, Fei S, Li L (2006). Isolation, characterization and evolutionary analysis of resistance gene analogs in annual ryegrass, perennial ryegrass and their hybrid. Physiologia Plantarum 126 (4): 627-638. - doi: 10.1111/j.1399-3054.20 06.00629.x

Li J, Ding J, Zhang W, Zhang Y, Tang P, Chen J-Q, Tian D, Yang $S$ (2010). Unique evolutionary pattern of numbers of Gramineous NBS-LRR genes. Molecular Genetics and Genomics 283 (5): 427-438. - doi: 10.1007/s00438-010-0527-6 Liu JJ, Ekramoddoullah AK (2003). Isolation, genetic variation and expression of TIR-NBSLRR resistance gene analogs from western 
white pine (Pinus monticola Dougl. ex. D. Don.). Molecular Genetics and Genomics 270: 432-441. - doi: 10.1007/s00438-003-0940-1

Liu JJ, Hunt R, Ekramoddoullah AK (2004). Recent insights into genetic resistance of western white pine to white pine blister rust. Recent Research Developments in Biotechnology and Bioengineering 6: 65-76. [online] URL: http://cfs.nrcan.gc.ca/publications?id=25421

Liu JJ, Ekramoddoullah AK, Hunt RS, Zamani A (2006). Identification and characterization of random amplified polymorphic DNA markers linked to a major gene $\left(\mathrm{Cr}_{2}\right)$ for resistance to Cronartium ribicola in Pinus monticola. Phytopathology 4: 395-399. - doi: 10.1094/PHYTO-960395

Liu JJ, Ekramoddoullah AK (2007). The CC-NBSLRR subfamily in Pinus monticola: targeted identification, gene expression, and genetic linkage with resistance to Cronartium ribicola. Phytopathology 97 (6): 728-736. - doi: 10.1094/ PHYTO-97-6-0728

Loehman RA, Clark JA, Keane RE (2011). Modeling effects of climate change and fire management on western white pine (Pinus monticola) in the northern Rocky Mountains, USA. Forests 2 (4): 832-860. - doi: 10.3390/f2040832

Lynn DJ, Higgs R, Gaines S, Tierney J, James T, Lloyd AT, Fares MA, Mulcahy G, Farrelly C (2004a). Bioinformatic discovery and initial characterisation of nine novel antimicrobial peptide genes in the chicken. Immunogenetics 56 (3): 170-177. - doi: 10.1007/s00251-004-0675-0 Lynn DJ, Lloyd AT, Fares MA, O'Farrelly C (2004b). Evidence of positively selected sites in mammalian a-defensins. Molecular Biology and Evolution 21 (5): 819-827. - doi: 10.1093/molbev/ msho84

Meyers BC, Kozik A, Griego A, Kuang H, Michelmore RW (2003). Genome-wide analysis of NBS-LRR-encoding genes in Arabidopsis. Plant Cell 15 (4): 809-834. - doi: 10.1105/tpc.009308 Mondragón-Palomino M, Meyers BC, Michelmore RW, Gaut BS (2002). Patterns of positive selection in the complete NBS-LRR gene family of Arabidopsis thaliana. Genome Research 12 (9): 1305-1315. - doi: 10.1101/gr.159402

Panetsos KP, Moulalis D, Mitsopoulos D, Skaltsoyiannes A (1983). Results of exotic and native forest species trials at the Aristotle University forests. Laboratory of Forest Genetics and Tree Breeding, Aristotle University, Publ. No. 6, Thessaloniki, Greece, pp. 30. [in Greek with English summary]

Panetsos KP, Alizoti PG, Moulalis D, Skaltsoyiannes A, Aravanopoulos FA (1996). Results of exotic forest species testing in Pertouli. In: Proceedings of the " 6 th Pan-Hellenic Conference of the Hellenic Scientific Society of Plant Genetics and Breeding". Florina (Greece) 2-4 Oct 1996. Hellenic Scientific Society for Plant Genetics and Breeding, Thessaloniki, Greece, pp. 310-316. [in Greek with English summary]

Papadima A, Sotikopoulos N (1995). Results of exotic and native forest species trials at the Aristotle University forests. BSc Thesis, Faculty of Agriculture, Forestry and Natural Environment, Aristotle University of Thessaloniki, Aristotle University Publ., Thessaloniki, Greece, pp. 73. [in Greek with English summary]

Parniske M, Hammond-Kosack KE, Golstein C, Thomas CM, Jones DA, Harrison K, Wulff BBH, Jones JDG (1997). Novel disease resistance specificities result from sequence exchange between tandemly repeated genes at the Cf4/9 locus of tomato. Cell 91 (6): 821-832. - doi: 10.1016/Soog2-8674(00)80470-5

Perazzolli M, Malacarne G, Baldo A, Righetti L, Bailey A, Fontana P, Velasco R, Malnoy M (2014). Characterization of resistance gene analogues (RGAs) in apple (Malus $x$ domestica Borkh.) and their evolutionary history of the Rosaceae family. PloS One 9 (2): e83844. - doi: 10.1371/journal.pone.0083844

Sekhwal MK, Li P, Lam I, Wang X, Cloutier S, You $F M$ (2015). Disease resistance gene analogs (RGAs) in plants. International Journal of Molecular Sciences 16 (8): 19248-19290. - doi: 10.3390/ijms160819248

Stamatakis A (2014). RAxML version 8: a tool for phylogenetic analysis and post-analysis of large phylogenies. Bioinformatics 30 (9): 1312-1313. doi: 10.1093/bioinformatics/btu033

Tamura K, Peterson D, Peterson N, Stecher G, Nei M, Kumar S (2011). MEGA5: molecular evolutionary genetics analysis using maximum likelihood, evolutionary distance, and maximum parsimony methods. Molecular Biology and
Evolution 28 (10): 2731-2739. - doi: 10.1093/mol bev/msr121

Wan H, Zhao Z, Malik AA, Qian C, Chen J (2010). Identification and characterization of potential NBS-encoding resistance genes and induction kinetics of a putative candidate gene associated with downy mildew resistance in Cucumis. BMC Plant Biology 10 (1): 186. - doi: 10.1186/14712229-10-186

Yang Z, Nielsen R (2000). Estimating synonymous and nonsynonymous substitution rates under realistic evolutionary models. Molecular Biology and Evolution 17 (1): 32-43. - doi: 10.1093/oxfordjournals.molbev.a026236

Yang Z (2007). PAML 4: phylogenetic analysis by maximum likelihood. Molecular Biology and Evolution 24 (8): 1586-1591. - doi: 10.1093/mol bev/msmo88

Yang S, Zhang X, Yue J-X, Tian D, Chen J-Q (2008). Recent duplications dominate NBSencoding gene expansion in two woody species. Molecular Genetics and Genomics 280 (3): 187-198. - doi: 10.1007/s00438-008-0355-0

Yang S, Li J, Zhang X, Zhang Q, Huang J, Chen JQ, Hartl DL, Tian D (2013). Rapidly evolving R genes in diverse grass species confer resistance to rice blast disease. Proceedings of the National Academy of Sciences USA 110 (46): 18572-18577. - doi: 10.1073/pnas.1318211110

Zambounis A, Elias M, Sterck L, Maumus F, Gachon CMM (2012). Highly dynamic exon shuffling in candidate pathogen receptors... What if brown algae were capable of adaptive immunity? Molecular Biology and Evolution 29 (4): 1263-1276. - doi: 10.1093/molbev/msr296

Zhou T, Wang Y, Chen JQ, Araki H, Jing Z, Jiang K, Shen J, Tian D (2004). Genome-wide identification of NBS genes in japonica rice reveals significant expansion of divergent non-TIR NBSLRR genes. Molecular Genetics and Genomics 271 (4): 402-415. - doi: 10.1007/s00438-004-09 90-z

\section{Supplementary Material}

Tab. S1 - YNoo method results of the $P$. monticola TIR-NBS-LRR RGAs.

Link: Zambounis_2050@supplo01.pdf 